Meta

Journal des traducteurs

Translators' Journal

\title{
La traduction des hyperonymes et autres termes de grande extension
}

\section{Danica Seleskovitch}

Volume 35, numéro 1, mars 1990

Actes du colloque international « La traduction proligère "

URI : https://id.erudit.org/iderudit/004083ar

DOI : https://doi.org/10.7202/004083ar

Aller au sommaire du numéro

Éditeur(s)

Les Presses de l'Université de Montréal

ISSN

0026-0452 (imprimé)

1492-1421 (numérique)

Découvrir la revue

Citer cet article

Seleskovitch, D. (1990). La traduction des hyperonymes et autres termes de grande extension. Meta, 35(1), 91-95. https://doi.org/10.7202/004083ar d'utilisation que vous pouvez consulter en ligne.

https://apropos.erudit.org/fr/usagers/politique-dutilisation/ 


\section{LA TRADUCTION DES HYPERONYMES ET AUTRES TERMES DE GRANDE EXTENSION}

DANICA SELESKOVITCH

Université de la Sorbonne Nouvelle-Paris III, Paris, France

Je commencerai cet exposé en rappelant la pièce de théâtre d'Ionesco La Leçon, qui s'est jouée à Paris pendant plusieurs dizaines d'années d'affilée.

Incapable d'apprendre le principe de la multiplication que son professeur s'acharne à lui enseigner, une élève trouve un jour non seulement la solution des petites opérations de quelques chiffres mais encore celle de nombres de six chiffres. Interloqué, le professeur lui demande d'expliquer cette extraordinaire performance pour s'entendre répondre qu'elle s'était résolue à apprendre toutes les solutions par cœur!

L'idée que se font certains de la traduction ressemble au rêve fou de Ionesco: connaître toutes les correspondances entre les lexiques de deux langues et savoir les règles du jeu morpho-syntaxique suffiraient pour traduire. Le caractère irréel de cette conception n'est plus à démontrer, chacun sait que les mots sont, comme les nombres, en quantité illimitée et qu'une bonne méthode est plus efficace que tous les savoirs appliqués en son absence. En traduction, connaître tous les mots d'un texte ne mène à rien si les méthodes de la traduction restent ignorées.

Rappelons tout d'abord cette observation d'E. Coseriu (1967):

On peut sans doute affirmer qu'Aristote, Hegel ou Heidegger ont su utiliser largement, pour leurs distinctions philosophiques, les ressources de la langue grecque et de la langue allemande, mais les distinctions en question ne sont pas des distinctions sémantiques de la «langue grecque» et de la «langue allemande» et ne peuvent être définies que par rapport à l'usage des trois philosophes: ce qu'on appelle le «langage de Heidegger» est, du point de vue lexicologique, en partie langue allemande, en partie terminologie philosophique générale et, en partie, terminologie spécifique heideggerienne.

Ce n'est pas seulement sous la plume d'un philosophe qu'un mot se charge de nuances de sens imprévisibles au niveau des langues; le phénomène est propre à la manifestation originale d'un vouloir-dire dans tous les discours.

Les sources de sens qui s'attachent, dans son emploi, à une structure sonore ou graphique sont multiples et la signification linguistique n'en est qu'une parmi d'autres. Il en découle que le traducteur doit non seulement connaître à fond deux langues, mais qu'il doit tenir compte du sens que prennent les signifiants dans le discours et choisir dans la langue d'arrivée le terme équivalent au sens, et non celui qui correspond à la signification.

Nous examinerons les incidences sur la traduction de la transformation en sens du sémantisme des mots lors de leur passage d'une langue à un discours en nous penchant sur une catégorie lexicale particulière: les mots dits «de grande extension mais de compréhension réduite» (Galisson et Coste : 1976).

Les hyperonymes ou termes génériques en sont un exemple typique; ce sont des têtes de séries lexicales dont les éléments successifs se font de plus en plus précis tout en désignant de moins en moins de choses; ainsi la série : végétal, plante, fleur, lilas ... On 
peut décrire la signification de «lilas» avec un luxe de détails que l'on serait bien en peine d'appliquer à «fleur» et plus encore à «plante» ou à «végétal».

Les hyperonymes sont intéressants pour le traductologue en ce qu'ils se substituent facilement dans le discours à des termes de moindre extension: «Je mets les fleurs dans le vase», dis-je en prenant les roses que j'ai ramenées du marché. Lorsque le style n'est pas en cause et que le contexte s'y prête, le traducteur peut les considérer comme synonymes. Il est un autre cas de terme de forte extension mais de compréhension réduite qui sollicite le traductologue de façon plus impérative; il s'agit de termes généraux qui voient se substituer en contexte une désignation à la signification qu'ils ont en langue. Le mot reste tel quel mais la chose désignée s'est précisée.

Jean Delisle (1984), par exemple, donne pour la traduction de pattern une liste de 66 équivalents provenant du fichier d'un traducteur de carrière; le mot anglais prenant dans chaque contexte un sens précis et à chaque fois différent exige une traduction chaque fois différente en français. Le traductologue relève là un phénomène qui l'intéresse au plus haut point car il signifie que le traducteur attentif à désigner la même chose que l'original ne peut pas employer de correspondance mais doit trouver un autre mot pour transmettre la désignation correcte dans l'autre langue. Les bons traducteurs désignent par un terme adéquat les multiples aspects d'une réalité couverte par un mot qui, s'il s'agissait de traduire la langue, appellerait une correspondance.

Nous avons mentionné le mot anglais «pattern»; mais l'anglais n'est pas différent des autres langues à cet égard; dans toutes, le passage de la signification linguistique d'un mot de grande extension à une désignation plus précise dans le discours impose au traducteur la même méthode : rechercher l'équivalence, ne pas se contenter de la correspondance au niveau linguistique.

Nous prendrons ici simplement deux exemples: un adverbe français, «pourquoi», et un substantif anglais, «utilities», pour montrer ce qu'est une traduction qui tient compte de l'actualisation des mots.

Le mot «pourquoi» dans la phrase «Pourquoi as-tu besoin de cet argent?» désigne une chose différente de celle qu'il désigne dans «Pourquoi ris-tu?». Le locuteur français actualise pourquoi une fois avec le sens de pour quoi faire, une autre fois avec le sens de pour quelle raison sans en prendre obligatoirement conscience dans la mesure où il utilise le même signifiant. Le locuteur allemand, par contre, explicite la spécificité de la notion désignée car sa langue l'a institutionnalisée. «Pourquoi ris-tu?» ne se traduit pas en allemand par Warum ..., mais bien par Weshalb lachst $D u$ ? et «Pourquoi as-tu besoin de cet argent ?» par Wozu brauchst Du das Geld? et non par Warum...

On dit souvent que les termes dits polysémiques contiennent des traits de signification dont le sujet parlant ne prend pas conscience au niveau de sa langue mais qui s'actualisent dans le discours. E. Coseriu (1981) préfère parler de «konfrontative Polysemie», contestant la notion de polysémie des mots à l'intérieur d'une même langue; il affirme, et notre pratique semble lui donner raison, qu'il n'existe qu'une polysémie opérationnelle, à savoir une multiplicité d'équivalences de désignations lors de la traduction des textes.

Le traductologue préfère cette explication à celle qui verrait dans l'emploi des mots une actualisation sémique. Il lui semble plus évident en effet que les termes de grande extension et de compréhension réduite se chargent de traits de signification grâce aux compléments cognitifs qu'apportent les éléments contextuels et situationnels du discours, cette charge donnant aux mots du discours des nuances qu'ils ne contiennent pas à l'origine. Ainsi «pourquoi» ne nous semble pas contenir des sèmes dont l'actualisation produirait le sens de wozu et de weshalb, il nous semble plutôt que, terme de grande extension et de compréhension réduite, il se charge de traits de signification grâce aux 
compléments cognitifs qu'apportent les éléments contextuels et situationnels du discours. Ainsi la signification «pour quoi faire» et «pour quelle raison» découlerait de l'ensemble des énoncés «Pourquoi as-tu besoin de cet argent?» et «Pourquoi ris-tu?» pour en charger pourquoi plutôt qu'elle ne serait une actualisation de sèmes préexistants.

Nous continuons notre démonstration en prenant le mot anglais «utilities». «Public utility» vise en anglais les entreprises de service public (distribution d'eau, de gaz, d'électricité). Voyons un texte ${ }^{1}$ où apparaît utilities, repérons la chose désignée et cherchons l'équivalence qui convient en traduction française.

Over a number of years, as far back as the mid-70s and more recently, the Commission (EEC) have just not been willing to accept that there is a flourishing market in electrical energy between utilities in Europe.

Le traducteur naif, qui ignore qu' on ne traduit pas un texte comme on traduit une langue, est tenté de traduire utilities par sa correspondance : service public, ce qui donnerait:

Un marché florissant de l'énergie électrique entre services publics européens.

Le bon traducteur, sachant qu'il faut traduire ce que dit le texte et non ce que dit la langue, traduira:

Les échanges d'énergie électrique entre les entreprises d'électricité européennes sont florissants.

Utilities a donc ici pour équivalent: «entreprises d'électricité».

Un autre exemple tiré du même texte confïme qu'il s'agit bien d'entreprises d'électricité.

Utilities have an obligation to provide a reliable and economic electricity supply to their customers

signifie de toute évidence:

Les entreprises d'électricité sont tenues d'assurer à leurs abonnés une distribution régulière d'électricité à un prix modéré.

Nul ne contestera que le contexte impose ici à «utilities» un sens de moindre extension mais de plus grande compréhension que n'est sa signification en anglais hors contexte et que le traducteur a eu raison de choisir en français le terme habituellement utilisé pour désigner la réalité que sont les entreprises d'électricité.

On pourrait allonger indéfiniment la liste des mots anglais de grande extension qui exigent la création d'équivalences pour désigner les mêmes réalités, pour désigner ce que nous appelons le sens. Malheureusement on s'efforce de plus en plus d'imposer maladroitement en français des correspondances telles que «maîtrise» pour control qui ne rendent service ni au français ni aux traducteurs, car elles restent tout aussi floues en contexte qu'au niveau linguistique, alors que control, tout comme pattern, exige en français autant d'équivalences que le texte confère de sens.

Les termes de grande extension sont des mots flous, indications sémantiques, non désignations d'une réalité ; ils ne se chargent pas seulement dans le discours de traits de signification supplémentaires, ils y perdent aussi une partie de leur potentiel de signification. Nous l'avons vu avec «utilities» qui perd dans le texte cité le pouvoir de désigner la distribution du gaz ou de l'eau.

Ayant dégagé dans notre livre (Seleskovitch et Lederer: 1989) le mécanisme réducteur du passage des mots de la langue en discours qui accompagne le gonflement de la 
signification devenue désignation de sens, nous n'y reviendrons pas ici. Nous insisterons par contre pour dire que le traducteur se doit de comprendre la distinction qu'il convient d'établir entre une langue et un discours. Il ne s'intéressera à la première que pour en perfectionner la connaissance alors qu'il placera le second au centre de l'opération qu'il réalise.

On peut extraire la nature générale de langues telles que le français, l'anglais, l'allemand, etc., en parlant de la langue comme Saussure ou de la compétence comme Chomsky. Mais la notion de langue, indépendante de toute situation de communication, est difficile à comprendre pour des non-linguistes et souvent confondue avec celle de discours, même par des linguistes qui oublient que si les textes ont des caractéristiques linguistiques qui leur sont propres, ils se chargent en outre de tous les compléments cognitifs que le lecteur apporte à leur rencontre et que l'auteur communiquait implicitement.

Tant qu'ils restent au niveau de la langue, les mots ont une forme, sonore ou graphique, à laquelle ne s'associe qu'un concept et rien d'autre.

Le concept (verbal) rassemble en une classe des éléments ayant des caractères communs, compte non tenu des différences qui peuvent exister entre eux (Galisson et Coste : 1976).

La façon la plus simple de comprendre la notion de concept verbal est de penser aux dessins enfantins (d'homme, de maison, d'arbre, etc.). Ces dessins

(...) rassemblent en une (présentation) des éléments ayant des caractères communs compte non tenu des différences qui existent entre eux.

On peut aussi penser, avec Alan Gardiner (1963), à comparer l'espèce à ses spécimens, l'espèce n'étant observable qu'à travers ses spécimens comme la langue n'est observable qu'à travers les discours faits dans cette langue, comme les concepts verbaux ne le sont qu'à travers leur emploi. La langue est conceptuelle; le mot qu'isolent les lexicologues, les phrases que construisent les grammairiens, sont concepts, aussi bien les mots «homme», «maison», «arbre», que la phrase : «Le chien est un animal carnivore à quatre pattes qui aboie.»

Le discours de son côté est un spécimen de l'espèce langue; malheureusement pour le traducteur, les formes de la langue ne changent pas toujours quand l'énonciation les charge de traits de signification supplémentaires. Le discours est unique et inédit; il est, dans une langue, le produit d'un individu à un moment donné, dans une situation donnée, adressé à un destinataire, singulier ou pluriel, qui partage le savoir (réel ou imaginaire) et les convictions et préjugés de l'auteur.

Aucun adulte ne pense au concept homme, arbre, maison quand on lui parle d'une maison, d'un homme ou d'un arbre; il y ajoute instantanément les compléments cognitifs qu'il tire de son expérience et du contexte. Le traducteur naîf ne fait pas de même, hélas. Il ignore que les mots du discours n'ont ni la même extension ni la même compréhension que les mots d'une langue; or, tous ceux qui savent peu ou prou deux langues traduisent; ignorant la différence entre une langue et un texte, ils restent des adeptes de la traduction des langues, que nous appelons transcodage. Aveuglés par des formes originales qui ne changent pas alors qu'elles désignent des réalités qui appellent d'autres formes dans une langue différente, ils se désolent et parlent d'intraduisibilité !

Le bon traducteur a conscience de la différence qui existe entre les concepts lexicaux et le lexique actualisé ; il choisit les formes qui conviennent dans la langue d'arrivée pour exprimer les sens du texte.

Il sait qu'on ne peut pas apprendre toutes les solutions par cœur; il sait par contre qu'on peut les trouver en appliquant des méthodes qui respectent le fonctionnement du 
langage. Il démontre par là même que les bonnes traductions constituent un poste d'observation de la vie du langage qui n'a pas son pareil. Il était utile que cela soit dit à un colloque sur la traduction proligère.

\section{NOTE}

1. Compte rendu de la réunion de l'Union internationale des producteurs et distributeurs d'électricité (UNIPEDE) du 11 février 1986.

\section{RÉFÉRENCES}

COSERIU, E. (1967) : «Structure lexicale et enseignement du vocabulaire», Les Théories linguistiques et leurs applications, Conseil de Cooperation Culturelle du Conseil de l'Europe, AIDELA.

COSERIU, E. (1981) : «Kontrastive Linguistik und Übersetzung im Verhältnis zueinander», Kühlwein, Thome, Wilss (eds.), Kontrastive Linguistik und Überseztungswissenschaft, München, Wilhelm Fink.

DELISLE, J. (1984): L'Analyse du discours comme méthode de traduction, Ottawa, Éditions de l'Université d'Ottawa.

GALISSON, R. et D. COSTE (1976) : Dictionnaire de didactique des langues, Paris, Hachette.

GARDINER, A. (1963): The Theory of Speech and Language, Oxford, Clarendon Press, (1 $1^{\mathrm{re}} \mathrm{ed} .:$ 1932).

SELESKOVITCH, D. et M. LEDERER (1989) : Pédagogie raisonnée de l'interprétation, Paris et Luxembourg, Didier Érudition et OPOCE. 